\section{Presence of Antitopoisomerase I Antibody Alone May Not Be Sufficient for the Diagnosis of Systemic Sclerosis}

\section{To the Editor:}

Systemic sclerosis (SSc) is characterized by altered immune function and vascular damage, which lead to extensive fibrosis. Experts underscore the importance of using SSc-specific autoantibodies to divide the disease into subsets for prognosis, management, and research ${ }^{1,2}$. One representative feature of the immunological abnormalities in patients with SSc is the presence of antinuclear antibodies (ANA) associated with autoantibody targets. The anticentromere (ACA), antitopoisomerase I (anti-topo I), anti-RNA polymerase I/III (ARA I/III), and anti-Th/To constitute about $80-85 \%$ of autoantibodies specific for SSc and can assist the physician in assessment ${ }^{3,4,5}$.

The 2013 classification criteria for SSc provide 3 points (toward a 9-point diagnosis) for patients who test positive for anti-ACA, anti-ARA III, or anti-topo I antibodies ${ }^{1}$. All 3 classical autoantibodies remain stable throughout the course of disease and tend to have a mutually exclusive association. While the presence of anti-topo I antibodies is thought to be highly specific and diagnostic for SSc, the significance of certain positive results remains unclear ${ }^{6,7}$.

Since the development of ELISA to detect anti-topo I antibodies, a number of different manual or automated immunoassays have become available to detect these antibodies ${ }^{8,9}$. The advantages of these alternative methods include the ease of use, the possibility of quantitative high-throughput analyses, and the consolidation of related tests in a single platform. From a clinical practice standpoint, in the absence of other clinical stigmata of SSc, the significance of the presence of the anti-topo I antibody results remains unclear. The purpose of our project was to determine the association with SSc of anti-topo I antibodies detected by multiplex testing for patients assessed during routine clinical evaluation at our institution.

Study participants included 3331 consecutive, unique patients from the University of Utah tested for anti-topo I antibodies at ARUP Laboratories from January 1, 2009, to March 5, 2017, as part of routine clinical laboratory investigations. Results for patients positive for anti-topo I antibodies (cutoff: $>41 \mathrm{AU} / \mathrm{ml}$-positive) had a retrospective chart review, and the 2013 American College of Rheumatology/ European League Against Rheumatism classification criteria for SSc were retrospectively applied. The study protocol was approved by the institutional review board (IRB \#00029507).

Anti-topo I testing was performed using the FIDIS Connective 10 multiplex bead assay (Theradiag). Antibody levels $>41 \mathrm{AU} / \mathrm{ml}$ were considered positive as per the manufacturer's recommendation. ANA were determined by indirect immunofluorescent antibody (IFA) using HEp-2 cell substrate (Inova Diagnostic) in some patients. For others, ANA was detected by ELISA (Bio-Rad) and positivity confirmed using HEp-2 cell substrate by IFA. Comparison between anti-topo I concentrations between groups was performed using Kruskal-Wallis test. The association of anti-topo I concentration and SSc was determined by logistic regression. The discrimination power of anti-topo I antibody was determined using receiver-operating characteristic curve (ROC) analysis.
A

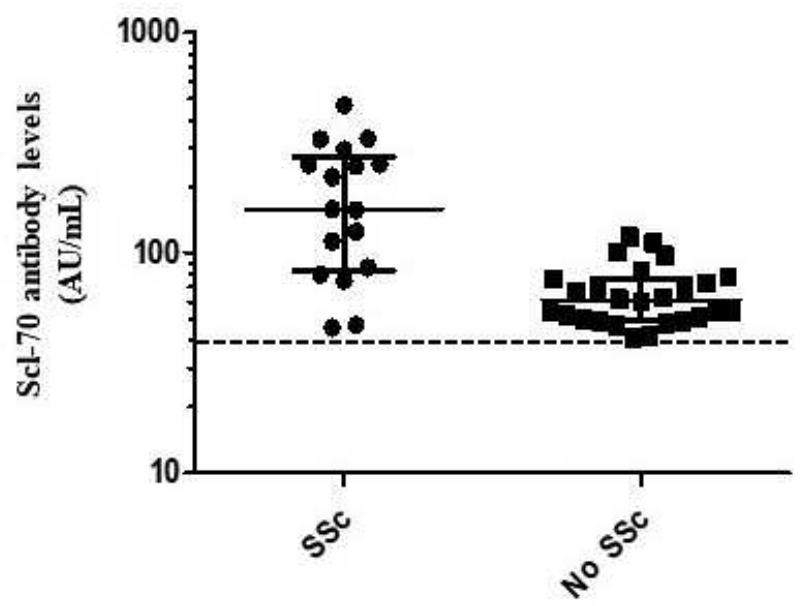

B

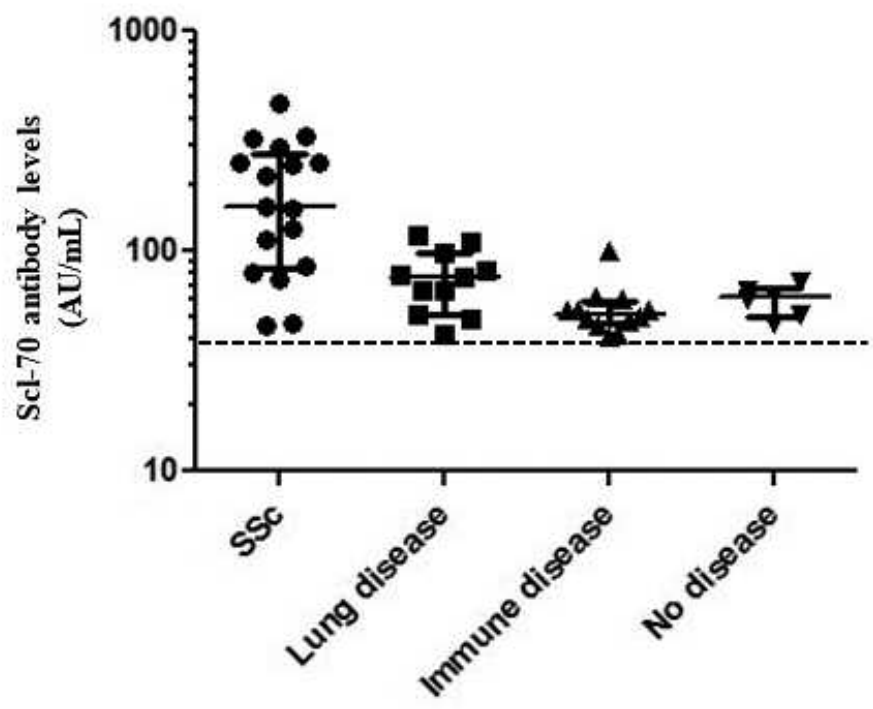

Figure 1. Clinical categorization of anti-topo I antibody-positive patients and their relative autoantibody titers. Anti-topo I antibody concentrations were significantly elevated in patients fulfilling the 2013 SSc criteria (panel A) compared to those who did not meet these requirements (panel B) - lung disease (bronchiectasis, empyema, tuberculosis, and usual interstitial pneumonia) and other immunemediated conditions (Sjögren syndrome, Graves' disease, inflammatory bowel disease, and sarcoid; $p$ = 0002). Anti-topo I antibody: antitopoisomerase I antibody; SSc: systemic sclerosis. 
Anti-topo I positivity was detected in $51(1.53 \%)$ of the 3331 patients. Of the 51 anti-topo I antibody-positive patients, 46 had complete clinical data available by chart review, and were categorized as SSc $(37 \%)$ or non-SSc $(63 \%)$. Non-SSc patients were individuals who did not meet criteria for SSc, but did include patients with lung disease (bronchiectasis, empyema, tuberculosis, and usual interstitial pneumonia) and other immune-mediated conditions (Sjögren syndrome, Graves' disease, inflammatory bowel disease, and sarcoid). None of these patients met criteria for interstitial pneumonia with autoimmune features at the time of chart review.

The median anti-topo I antibodies were significantly elevated in SSc compared to non-SSc patients $(\mathrm{p}=0.0002$; Figure 1). No difference in median antibody level was observed in the subsets of patients with non-SSc. All patients with SSc were ANA antibody-positive compared to only $46.4 \%$ of non-SSc subjects $(\mathrm{p}<0.001)$. Using logistic regression analysis, anti-topo I antibody level of about $125 \mathrm{AU} / \mathrm{ml}$ was predictive of SSc (Figure 2) with an estimated area under the ROC of 0.8641 (data not shown), sensitivity of $65 \%$, and specificity of $100 \%$.

Although retrospective in design, our investigation has a few strengths with opportunities for future in-depth consideration concerning interpretation of anti-topo 1. The importance of a positive result should be evaluated with a pretest possibility; however, in our cohort we did not have the details on why the test was ordered. Nonetheless, this accurately reflects clinical practice in which autoantibody testing is not restricted to rheumatologists ${ }^{10}$. The low number of cases highlights the need for concerted efforts in not only clinical research, but also establishing reagents to optimally categorize patients as new technologies emerge.

Our study suggests that the presence of an ANA by IFA and significantly elevated titers of anti-topo 1 is supportive of a diagnosis of SSc, and that the presence of anti-topo I antibody alone is not sufficient for the diagnosis of SSc. Further, in ANA-negative patients with low titer anti-topo 1, other lung diseases may be present. This finding warrants further research into the pathogenesis of anti-topo 1 antibodies in the setting of pulmonary epithelial damage.

ANNE E. TEBO, PhD, Department of Pathology, University of Utah, and ARUP Laboratories; ROBERT L. SCHMIDT, MD, PhD, MBA, Department of Pathology, University of Utah, and ARUP Laboratories; TRACY M.

FRECH, MD, Department of Rheumatology, University of Utah, Salt Lake City, Utah, USA. Address correspondence to Dr. T.M. Frech, University of Utah, Internal Medicine, 30 N. 1900 E., Salt Lake City, Utah 84132, USA. E-mail: tracy.frech@hsc.utah.edu

\section{REFERENCES}

1. van den Hoogen F, Khanna D, Fransen J, Johnson SR, Baron M, Tyndall A, et al. 2013 classification criteria for systemic sclerosis: an American College of Rheumatology/European League against Rheumatism collaborative initiative. Arthritis Rheum 2013;65: 2737-47.

2. Johnson SR, Soowamber ML, Fransen J, Khanna D, Van Den Hoogen F, Baron M, et al. There is a need for new systemic sclerosis subset criteria. A content analytic approach. Scand J Rheumatol 2018;47:62-70.

3. Cepeda EJ, Reveille JD. Autoantibodies in systemic sclerosis and fibrosing syndromes: clinical indications and relevance. Curr Opin Rheumatol 2004;16:723-32.

4. Gabrielli A, Avvedimento EV, Krieg T. Scleroderma. N Engl J Med 2009;360:1989-2003.

5. Patterson KA, Roberts-Thomson PJ, Lester S, Tan JA, Hakendorf P, Rischmueller M, et al. Interpretation of an extended autoantibody profile in a well-characterized Australian systemic sclerosis (scleroderma) cohort using principal components analysis. Arthritis Rheumatol 2015;67:3234-44.

6. Nandiwada SL, Peterson LK, Mayes MD, Jaskowski TD, Malmberg $\mathrm{E}$, Assassi S, et al. Ethnic differences in autoantibody diversity and hierarchy: more clues from a US cohort of patients with systemic sclerosis. Rheumatol 2016;43:1816-24.

7. Jarzabek-Chorzelska M, Blaszczyk M, Jablonska S, Chorzelski T, Kumar V, Beutner EH. Scl-70 antibody - a specific marker of systemic sclerosis. Brit J Dermatol 1986;115:393-401.

8. Vazquez-Abad D, Tian L, Zanetti M, Rothfield NF. A crossreactive idiotype in scleroderma. Clin Exp Immunol 1997;108:420-7.

9. Bridges AJ, Lorden TE, Havighurst TC. Autoantibody testing for connective tissue diseases. Comparison of immunodiffusion, immunoblot, and enzyme immunoassay. Am J Clin Pathol 1997;108:406-10

10. Albon S, Bunn C, Swana G, Karim Y. Performance of a multiplex assay compared to enzyme and precipitation methods for anti-ENA testing in systemic lupus and systemic sclerosis. J Immunol Methods 2011;365:126-31.

First Release March 1 2019; J Rheumatol 2019;46:4; doi: $10.3899 /$ jrheum.180503

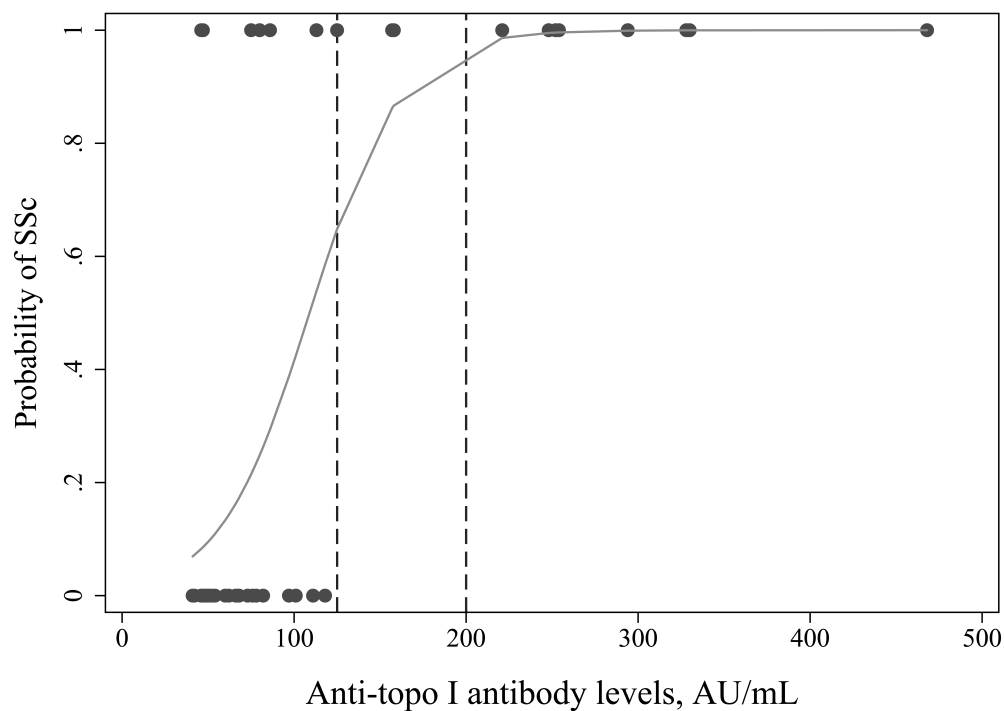

Figure 2. Anti-topo I antibody levels may be useful in predicting risk for SSc. Using logistic regression, an estimated probability of 1 for SSc was attained at anti-Scl-70 antibody level of about $125 \mathrm{AU} / \mathrm{ml}$. Anti-topo I antibody: antitopoisomerase I antibody; SSc: systemic sclerosis. 